\title{
DIMENSIONES DEL PERFIL DOCENTE EN LA ERA GLOBAL
}

rev.ciencia.poder.aéreo.6: $11-16,2011$

Autor $^{1}$

David E. López Cortés

Fecha recepción: 9 de Junio de 2011 Fecha aceptación: 15 de Julio de 2011.

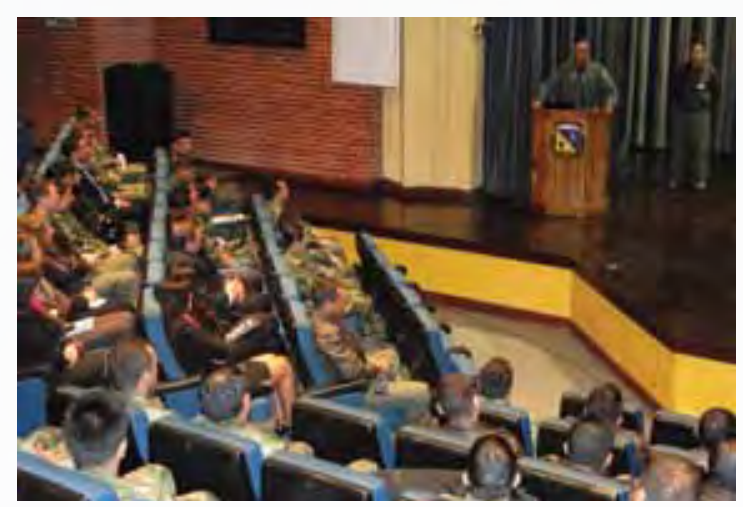

\section{Resumen}

En este texto se abordarán las nuevas competencias y retos de los educadores en el ejercicio de su profesión, frente a la globalización en la sociedad del conocimiento, y en el contexto del surgimiento de las Tecnologías de la Información y Comunicación (TIC) como herramienta didáctica de enseñanza que genere aprendizajes con sentido en el salón de clase; todo lo cual exige docentes que relacionen los conocimientos y las competencias en el entorno cotidiano de los estudiantes, a través de experiencias de aula inmersas en ambientes educativos de aprendizaje, investigación, creatividad, innovación y solución de problemas del entorno natural, social y creado.
Palabras Clave: Competencia, docente, globalización, sociedad del conocimiento, tecnologías de la información y comunicación (TIC), enseñanza, didáctica.

\section{Abstract}

This text will address the new responsibilities and challenges educators have in their profession facing globalization in a knowledge society, and according to the new Information and Communication Technologies (ICTs) as a didactic teaching tool in order to generate meaningful learning in the classroom. All of this requires professors connect students knowledge and skills in their daily environment through classroom experiences embedded in educational learning atmospheres, research, creativity, innovation and natural, social and created environment problem solving.

Key Words: Competition, teaching, globaliztion, knowledge society, information technology and communication (ICT), education, teaching.

\section{Introducción}

El presente artículo, es una breve exploración de las implicaciones del cambio producido por la globaliza-

${ }^{1}$ Abogado y Especialista en Derecho Público de la Universidad Libre, Especialista en Educación Ambiental y Desarrollo Comunitario de la Universidad de Cundinamarca, licenciado en Biología de la Universidad Pedagógica Nacional, actualmente docente del Instituto Militar Aeronáutico, IMA. Correo electrónico: biologiadavid@yahoo.es 


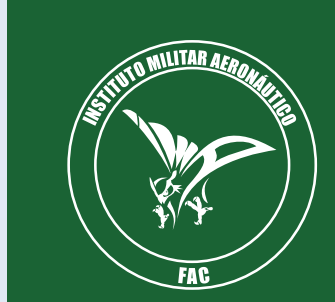

Ciencia \&

Educación

ción y el surgimiento de nuevas competencias en el ejercicio de la profesión docente, así como el uso de las Tecnologías de la Información y Comunicación (TIC) en la sociedad del conocimiento como factor de productividad y competitividad en la economía mundial y especialmente en el entorno nacional. Como se reconoce ampliamente, el cumplimiento satisfactorio de las metas planteadas por la política educativa colombiana solo es posible si se construye una educación de calidad, de alto desempeño en competencias laborales, que cierre las brechas de la inequidad, pobreza y desequilibrio social; incorporando en esta construcción, procesos de formación que hacen uso de tecnologías de la educación, acompañados de valores que aseguren mejorar la calidad de vida y la convivencia pacífica de todos los colombianos, donde el educador tiene un papel protagónico.

\section{Dimensiones del Perfil Docente en la Era Global}

La sociedad contemporánea, en el contexto socioeconómico de la globalización, se encuentra inmersa en un proceso de modernización constante en el cual priman la tecnología, la comunicación y la información. En este sentido, se constituye una sociedad centrada en torno a las tecnologías de la información, las cuales conllevan cambios que alcanzan todos los ámbitos de la actividad humana y de la enseñanza induciendo a que el docente desarrolle nuevas competencias en su actividad profesional, en el uso de didácticas informáticas en la construcción del conocimiento.

Estas características de la sociedad del internet modifican radicalmente la forma en que se dan las relaciones sociales, económicas, políticas, culturales y redefiniendo los sistemas económicos, los modelos de producción, el mercado, el trabajo, la educación, el consumo, entre otros aspectos.

Esta revolución tecnológica genera novedosas formas de organización social y productiva, que en términos de Castells (2002) es denominada como Sociedad Red, pensada como una estructura interactiva para la transmisión de información y conocimiento. Así pues, la educación no escapa a los efectos de la globalización en la que están enfrentados todos los países del mundo, que ha originado un punto de quiebre en los sistemas económicos y productivos, cuya riqueza no está proporcionada por mercancías tradicionales, sino que está marcada por la capacidad de producir bienes y servicios cada vez más sofisticados, con valores agregados, los cuales se logran adicionando conocimiento, innovación, creatividad y tecnologías tanto en los procesos industriales como educativos.

Frente a estos retos y ante la necesaria globalización, el sistema educativo colombiano, se hace ineludible crear una simbiosis entre los centros de educación superior y el sector productivo que permita hacer frente a la dinámica educativa y económica de este siglo, y en la gestación de esta transformación, los docentes se constituyen en elementos centrales.

Los educadores, como le puede pasar a un buen número de personas, distraídamente, no se han percatado -en su gran mayoría- del alcance del cambio producido por la globalización: emergen nuevas competencias y es indispensable preparar docentes que respondan a ellas. La pregunta que surge es ¿están los educadores verdaderamente preparados para enfrentar este nuevo reto y lo pueden administrar realmente en su ejercicio profesional?

Es claro que la sociedad del conocimiento pone en juego nuevas presiones sobre el sistema educativo y en especial en la educación superior, los cuales a su vez, suelen recaer especialmente en los educadores como piezas fundamentales en el engranaje transformador de la educación en la formación del nuevo modelo de ser humano que requiere el siglo XXI.

La educación es prioritaria en el mejoramiento de las condiciones y calidad de vida de los hombres y mujeres por cuanto provee las herramientas esenciales para participar en el cambio transformacional en los medios de producción y la creación de empleo. En tal 
contexto el reto es favorecer el manejo y la creación de tecnologías complejas, la capacidad de adaptación y la competitividad por parte de la población activa, con el propósito de que trascienda el modelo educativo asentado durante mucho tiempo en la sociedad manufacturera y consumista de todo lo extranjero, que ha creado una dependencia económica externa como se observa aún hoy día, con el interés puesto en la consecución de tratados de libre comercio con múltiples países del globo.

Si se mejora la educación, es posible liberarse de la mencionada dependencia económica y tecnológica.

El uso de las nuevas Tecnologías de la Información y Comunicación (TIC) ha sido la puerta de entrada a los nuevos paradigmas que el profesor ha tenido que asumir, para lo cual ha tenido que autoformarse en ellas como herramientas de trabajo en su rol protagónico de guía y acompañante en la construcción del conocimiento; para este desempeño, se obliga a trascender de la disciplina que está enseñando, conocer otras formas de ilustración, saber usarlas con propiedad, relacionarlas con lo que siempre ha hecho, pero hacerlo mejor, empleando la tecnología en el aula que implica actividades adicionales que no formaban parte de sus tareas habituales, afectando así su pergamino protagónico en la enseñanza, perdiendo el papel centrado en su figura y sus conocimientos que debe ir incorporándolo en su actividad mediadora con el educando; de ahí que es trascendente el aprender a aprender del docente en su desarrollo profesional.

El profesor como poseedor de saberes compromete el tener conocimientos profundos y fuertes del quehacer propio de su disciplina, de los saberes pedagógicos, psicológicos, didácticos, epistemológicos e históricos como mínimo vital de su área de conocimiento, para poder ejercitar la docencia con idoneidad, y ser motor de conocimiento y desarrollo que le posibilite el crecimiento académico de sus estudiantes, interactuar social y cultural en la sociedad del conocimiento; siendo investigador de la actividad educativa en todas las dimensiones de su trabajo magisterial, mejorando los métodos de enseñanza, innovando el currículo, la evaluación y ampliando las herramientas didácticas en sus clases.

Consciente de ello, UNESCO en la Conferencia Mundial de 1998 sobre educación superior, "desarrollo profesional del personal universitario: una misión continua" definió algunas características, actitudes y aptitudes necesarias en el docente para el siglo XXI, entre las que se encuentra:

Emplear las posibilidades que brinda el desarrollo de las tecnologías de información y la comunicación en el proceso educativo (UNESCO, 1998).

A nivel local, diversas universidades colombianas vienen desarrollando propuestas de formación de docentes incorporando TIC; estos esfuerzos se han iniciado como programas cortos y puntuales (los más comunes), a programas institucionales.

Una aproximación al respecto es la propuesta por Echeverría (1996), quien descompone las competencias profesionales desde los pilares de la educación indicados por la UNESCO, de la siguiente manera:

a) Competencia Técnica (saber): tener los conocimientos especializados que permitan dominar, como experto, los contenidos y las tareas vinculadas a la propia actividad laboral.

b) Competencia Metodológica (saber hacer): aplicar los conocimientos a situaciones laborales concretas utilizando los procedimientos adecuados, solucionar problemas de manera autónoma y transferir las situaciones adquiridas a situaciones novedosas.

c) Competencia Participativa (saber estar): atender el mercado laboral, predisposición al entendimiento interpersonal, así como a la comunicación y cooperación con los otros demostrando un comportamiento orientado al grupo. 


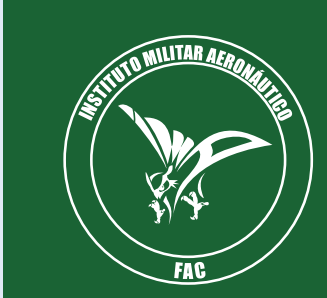

Ciencia \&

Educación

d) Competencia Personal (saber ser): tener una imagen realista de uno mismo, actuar de acuerdo con las propias convicciones, asumir responsabilidades, tomar decisiones y relativizar posibles frustraciones.

Desde esta perspectiva, las competencias no son potencialidades innatas del ser humano, sino que forman parte de una construcción permanente de quien aprende, desde una clara intencionalidad de producir o desempeñar una tarea específica de manera eficiente y eficaz.

Las diversas posturas frente al término competencia se aproximan a un concepto holístico como la capacidad productiva de un individuo (docente) que se define y mide en términos de desempeño en un determinado contexto laboral (educativo), y no solo de conocimientos, habilidades o destrezas en abstracto; es decir, la competencia es la integración entre el saber, el saber hacer, el saber ser y el saber convivir.

Son los docentes de educación superior con el apoyo decidido de los centros universitarios, los llamados a desarrollar y potenciar una serie de competencias deseables para lograr el adecuado desempeño de sus funciones en los nuevos entornos de aprendizaje. Para ello, es importante establecer un microcurriculum que desarrolle en los docentes universitarios el uso de las nuevas tecnologías de la información en componentes básicos, del cual hace parte el desarrollo de habilidades tecnológicas como eje transversal de los otros componentes del modelo educativo, pedagógico, didáctico y metodológico del docente de este siglo.

Es así, como a partir de este componente tecnológico se ha de generar una primera estructura, a la luz de los intereses, necesidades y tendencias de la educación superior: los conceptos fundamentales, el conocimiento, las destrezas y actitudes en el uso de TIC permitirán al docente ser competitivo en su trabajo.

Adicionalmente, la UNESCO tiene unos criterios que están agrupados en categorías básicas, las cuales se subdividen en los pilares fundamentales de la educación propuestos por Delors (1996). Estas categorías de base son: alfabetización tecnológica, desarrollo productivo, integración TIC en ambientes de aprendizaje y educación en ambientes virtuales, las cuales implican desde el uso instrumental de las herramientas, hasta llegar a procesos de incorporación de las mismas en actividades de enseñanza-aprendizaje, en un continuo que va desde la preespecialidad hasta la virtualidad.

Estas inician con el conocimiento de los conceptos asociados a las TIC (saber-saber), luego centran su atención en los procedimientos, como el desarrollo de habilidades y destrezas asociadas al uso de las TIC (saber - hacer) y por último, resalten un elemento esencial en la configuración de estas competencias para docentes desde las actitudes frente a las TIC (saber - ser) y frente a la actitud hacia el trabajo colaborativo mediado con TIC (saber - convivir).

Los nuevos escenarios de la sociedad de la información requieren repensar los perfiles de conocimiento de las personas que demandan estos entornos. En este sentido, es importante hacer explícitos los aportes de las TIC en la educación, realizando las adaptaciones curriculares que permitan su efectiva inclusión al ámbito universitario. De allí que los docentes se constituyen en un elemento primordial en la integración de las TIC, siendo necesario que éstos desarrollen las competencias que les permitan aprovecharlas en las diferentes asignaturas que orientan.

Para el desarrollo de competencias deseables de un docente que genere cambio en los métodos de enseñanza y deje atrás el modelo tradicional clásico de instrucción, que desafortunadamente muchos profesores aún practican, es imprescindible distinguir las semejanzas y diferencias de uno y otro paradigma; para ello se presenta la Tabla No. 1, en la que se especifican las características y tareas del maestro desde dos modelos educativos contrapuestos: 
Tabla No. 1 Modelo Tradicional vs. Modelo Tecnológico

Modelo tradicional o Clásico

1. Profesor como instructor.

2. Se pone el énfasis en la enseñanza.

3. Profesor aislado.

4. Suele aplicar los recursos sin diseñarlos.

5. Didáctica basada en la exposición y con carácter unidireccional.

6. Sólo la verdad y el acierto proporcionan aprendizaje.

7. Restringe la autonomía del alumno.

8. El uso de nuevas tecnologías está al margen de la programación.

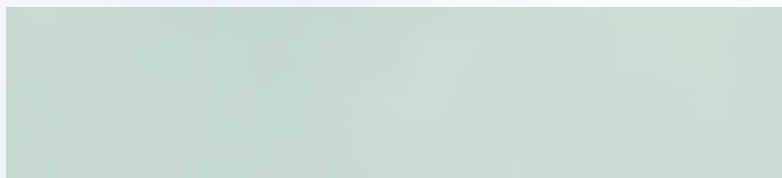

\section{Modelo Tecnológico}

1. El profesor como mediador.

2. Se pone el énfasis en el aprendizaje.

3. El profesor colabora con el equipo docente y forma redes de apoyo.

4. Diseña y gestiona sus propios recursos.

5. Didáctica basada en la investigación y con carácter bidireccional.

6. Utiliza el error como fuente de aprendizaje.

7. Fomenta la autonomía del alumno.

8. El uso de nuevas tecnologías está integrado en el curriculum. El profesor tiene competencias básicas en TIC.

9. Es investigador, innovador, creativo, transformador de su entorno y de la realidad de sus estudiantes.

(Adicionado por David E. López C)

Información tomada con base en el trabajo presentado por Cabrero y Ggisbert (2008), p.13.

\section{Conclusiones}

En este marco, y a partir de las competencias básicas antes expuestas que debe tener todo docente, se puede concluir que el educador, además del dominio de la materia que imparte, debe estar acompañado de competencia cultural, cualidades pedagógicas (habilidades didácticas, tutoría, técnicas de investigación -acción, conocimientos psicológicos y sociales), habilidades instrumentales y conocimientos de nuevos lenguajes, características personales (madurez, seguridad, autoestima, equilibrio emocional, empatía) y una constante actualización de su actividad docente. Así mismo, se sugiere que las instituciones de educación superior con la necesidad de la acreditación de sus programas de alta calidad, deben contar con docentes mínimo con maestría; por esto es importante apoyar a los educadores con becas y préstamos para realizar estudios de doctorado, creando y desarrollando así centros de investigación que brinden soluciones a las necesidades del país, capacitación permanente en TIC que permitan mejorar las didácticas de enseñanza y las competencias académicas a los docentes y responder así a los nuevos retos que trae la sociedad globalizada del conocimiento.

Por último, es necesario comentar que el actual contexto se convierte en una excelente oportunidad para que el maestro esté atento a todas las posibilidades de comunicación que el medio le ofrece, pueda ha- 


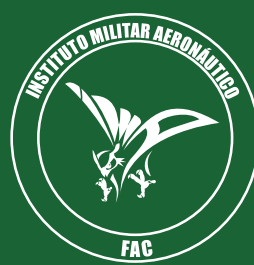

Ciencia \&

Educación

Revista Ciencia y Poder Aéreo. Edición No 6 - Septiembre 2011 ISSN 1909 - 7050

cer más adecuado, exitoso y atractivo el proceso de aprendizaje de sus estudiantes; un profesional de la educación que revise críticamente su propia práctica desde la reflexión de sus intervenciones como constructor de la educación, que pueda ayudar a sus alumnos a aprender a aprender en una sociedad cambiante y en constante evolución.

La tarea del pedagogo se dirige a que los estudiantes aprendan por ellos mismos, y para lograr este propósito se debe realizar bajo el lema "aprender haciendo", elaborando numerosos trabajos prácticos de exploración e investigación, que conduzcan a la solución de problemas de su entorno, que permitan formar un educando innovador, creativo y transformador de su ambiente natural, social, creado y específico; aportando a las necesidades del país, asentado en bases pedagógicas y didácticas de la enseñanza, con conciencia social y política que proyecte la figura del docente como facilitador de ideas, sueños y proyectos como aquel educador capaz de preparar a las nuevas generaciones que producirán el cambio científico, tecnológico y productivo en la era global en la que el país debe ser competitivo en la economía mundial.

\section{Referencias}

Cabrero, J. (2008). Un referente básico para la formación en Internet Educación y tecnologías de la información y la comunicación. Paradigmas teóricos de la investigación. Colombia. Editorial Magisterio.

Castells, M. (2000). The rise of network society. Vol.2. The information Age: Economy, Society and culture. Oxford Malden, M A; Blackwell Publisher.

Delors, J. (1996). La educación encierra un tesoro: informe a la Unesco de la comisión internacional sobre educación para el siglo XXI. Ediciones Unesco.

Echeverría, B. (1996). Formación Profesional. Barcelona: PPU

Evolución de la World Wide Web y nuevos desafíos a los que debe enfrentarse la Investigación en la Universidad. (s.f.). Disponible en:http://renia.cujae.edu.cu/index.php/revistacientifica/ar ticle/view/45/pdf_6

International Society for Technology in Education. ISTE: NETS for Students. Disponible en: http://www.iste.org/.

UNESCO (1998). Las posibilidades que brinda el desarrollo de las tecnologías de información y la comunicación en el proceso educativo. Disponible en: http://unesdoc.unesco.org/ images/0019/001912/191218s.pdf

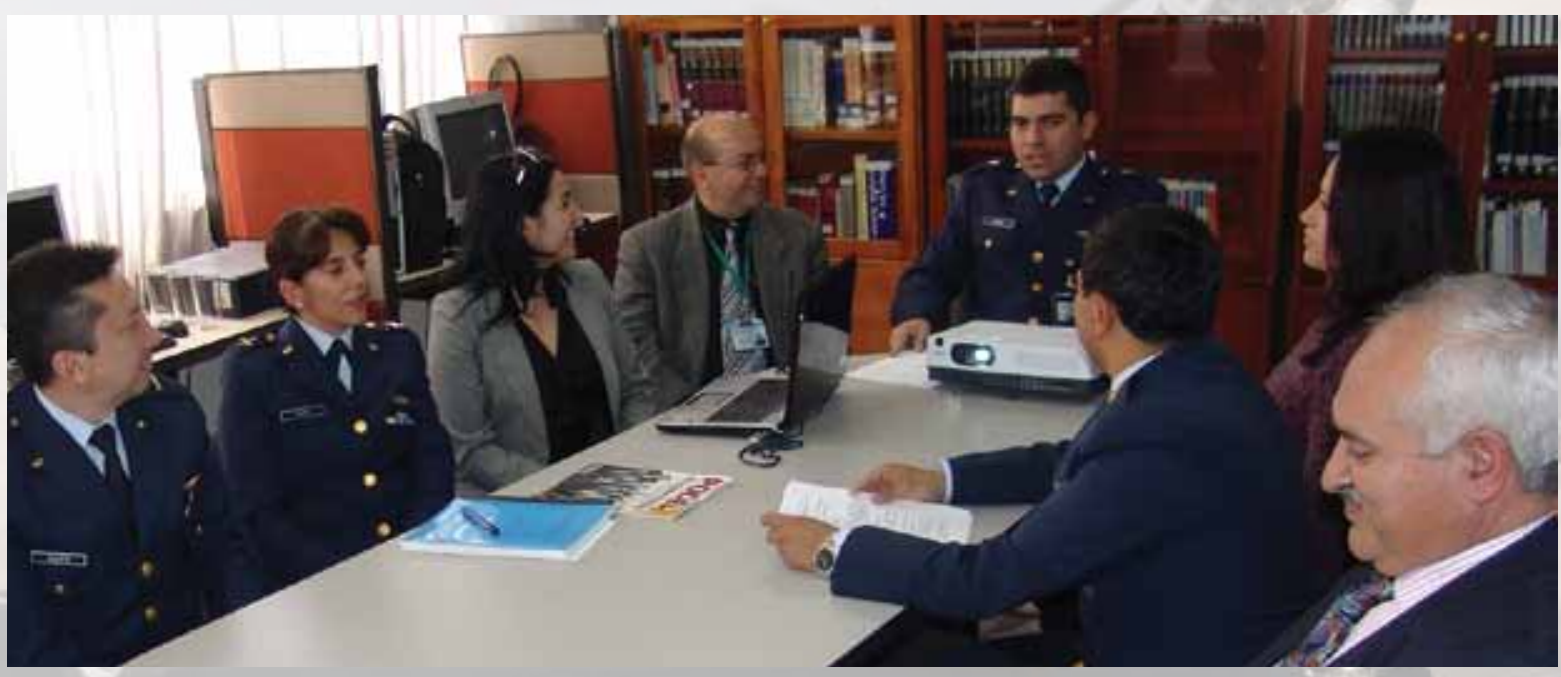

